\title{
Genetic Risk for Psychiatric Disorders and Telomere Length
}

\begin{abstract}
Alish B. Palmos', Gerome Breen 1,2, Laura Goodwin ${ }^{3,4}$, Souci Frissa ${ }^{5}$, Stephani L. Hatch ${ }^{5}$, Matthew Hotopf ${ }^{2,3,6}$, Sandrine Thuret ${ }^{7}$, Cathryn M. Lewis ${ }^{1,2}$ and Timothy R. Powell ${ }^{1 *}$

1 Social, Genetic and Developmental Psychiatry Centre, Institute of Psychiatry, Psychology and Neuroscience, King's College London, London, United Kingdom, ${ }^{2}$ National Institute for Health Research Biomedical Research Centre for Mental Health, Institute of Psychiatry, Psychology and Neuroscience, Maudsley Hospital, King's College London, London, United Kingdom, ${ }^{3}$ Department of Psychological Medicine, Institute of Psychiatry, Psychology \& Neuroscience, King's College London, London, United Kingdom, ${ }^{4}$ Department of Psychological Sciences, University of Liverpool, Liverpool, United Kingdom, ${ }^{5}$ Health Service \& Population Research Department, Institute of Psychiatry, Psychology \& Neuroscience, King's College London, London, United Kingdom, ${ }^{6}$ South London and Maudsley NHS Foundation Trust, London, United Kingdom, ${ }^{7}$ Department of Basic and Clinical Neuroscience, Institute of Psychiatry, Psychology and Neuroscience, King's College London, London, United Kingdom
\end{abstract}

Background: Previous studies have revealed associations between psychiatric disorder diagnosis and shorter telomere length. Here, we attempt to discern whether genetic risk for psychiatric disorders, or use of pharmacological treatments (i.e., antidepressants), predict shorter telomere length and risk for aging-related disease in a United Kingdom population sample.

Methods: DNA samples from blood were available from 351 participants who were recruited as part of the South East London Community Health (SELCoH) Study, and for which whole-genome genotype data was available. Leukocyte telomere length was characterized using quantitative polymerase chain reactions. Individualized polygenic risk scores for major depressive disorder (MDD), bipolar disorder (BD), and schizophrenia (SCZ) were calculated using Psychiatric Genomics Consortium summary statistics. We subsequently performed linear models, to discern the impact polygenic risk for psychiatric disorders (an etiological risk factor) and antidepressant use (common pharmacological treatment) have on telomere length, whilst accounting for other lifestyle/health factors (e.g., BMl, smoking).

Results: There were no significant associations between polygenic risk for any of the psychiatric disorders tested and telomere length $(p>0.05)$. Antidepressant use was significantly associated with shorter telomere length and this was independent from a depression diagnosis or current depression severity $(0 \leq 0.01)$. Antidepressant use was also associated with a significantly higher risk of aging-related disease, which was independent from depression diagnosis ( $p \leq 0.05$ ).

Conclusion: Genetic risk for psychiatric disorders is not associated with shorter telomere length. Further studies are now needed to prospectively characterize if antidepressant use increases risk for aging-related disease and telomere shortening, or whether people who age faster and have aging-related diseases are just more likely to be prescribed antidepressants.

Keywords: polygenic risk score, psychiatry, antidepressants, aging, telomeres 


\section{INTRODUCTION}

The complex and dynamic relationship between physical illness and psychiatric disorders was highlighted in a Chief Medical Officer's 2013 annual report, which stated that people with a psychiatric disorder experience worse physical health than those without (Davies, 2014). The comorbidity of a long-term physical illness and psychiatric disorder raises total health care costs by at least $45 \%$ per person (Naylor et al., 2012), and increases the risk of early mortality (Chang et al., 2011).

Psychiatric disorders such as major depressive disorder (MDD), bipolar disorder (BD), and schizophrenia (SCZ) have all been linked to an increased risk of severe medical conditions throughout a person's life (Kessler et al., 2005; Rai et al., 2014; Kang et al., 2015; Menear et al., 2015). The prevalence of MDD in patients with a physical illness is reported to be around twofold to threefold higher than in the general population and comorbid illnesses such as diabetes, pain, cancer, stroke, and cardiovascular disease have become an increasingly important global health issue (Kessler et al., 2005; Kang et al., 2015; Winkler et al., 2015). A cross-sectional report on patients with schizophrenia has stated that more than $50 \%$ of patients with schizophrenia possess at least one comorbid physical illness such as chronic pain, liver disease, and in particular, type-2 diabetes, which can often lead to microvascular and macrovascular complications such as neuropathy, coronary heart disease, and stroke (Chwastiak et al., 2006; Meeuwisse-Pasterkamp et al., 2008; Smith et al., 2013). BD is also associated with medical conditions likely to increase mortality, including respiratory, cardiovascular, and endocrine problems (Kemp et al., 2010; Forty et al., 2014).

Interestingly, medical conditions that show the highest prevalence of comorbidity across these three psychiatric disorders (MDD, $\mathrm{BD}$, and $\mathrm{SCZ}$ ) tend to be associated with aging; namely cardiovascular disease, stroke, obesity, and type-2 diabetes. This suggests that psychiatric disorders may be associated with faster biological aging, and some studies which assay 'telomeres' support this notion (e.g., Simon et al., 2006).

Telomeres are found on the ends of chromosomes and are special structures that are essential for protecting the chromatin from DNA damage during recombination (de Lange, 2002). Telomeres get shorter with each cell division as a result of the end-replication problem and this progressive telomere shortening is thought to represent a 'molecular clock' which underlies cell aging (Blackburn, 2001; Collins and Mitchell, 2002). Telomere shortening to a critical length, results in the realization of the "Hayflick limit" and a reduction in the ability of cells to divide (Blackburn, 2001). Ultimately, this means that new cells are less able to replace old, damaged cells, and thus the body becomes more vulnerable to aging-related diseases (Blackburn, 2001; Samani et al., 2001; Collins and Mitchell, 2002; Oh et al., 2003; Blasco, 2005).

Shorter telomeres have been demonstrated in patients suffering from MDD, BD, and SCZ compared to controls, leading to speculation that telomeres may play a role in psychiatric disorder etiology (Simon et al., 2006; Lung et al., 2007; Kao et al., 2008; Yu et al., 2008; Hartmann et al., 2010;
Elvsåshagen et al., 2011; Wikgren et al., 2012; Lima et al., 2015; Lindqvist et al., 2015; Mamdani et al., 2015). However, understanding whether or not telomere shortening is directly related to the pathophysiology of psychiatric disorders is difficult to determine from classic case-control studies due to confounding factors. Specifically, psychiatric patients recruited to case-control studies are often already taking medications and are significantly more likely to be leading unhealthy lifestyles (e.g., poor diet, smoking), which may impact upon rates of telomere shortening. Subsequently, what may be more informative is to study a known causative risk mechanism for a psychiatric disorders and its association with telomere length, outside the context of the disorder itself and associated environments polygenic risk scores (PRS) represent one option which may allow us to achieve this.

Polygenic risk scores represent the cumulative effect of many common risk variants for a given trait and are an effective way of quantifying genetic risk for a psychiatric disorder, even in non-clinical population cohorts (Sullivan, 2010; Clarke et al., 2016). PRS have previously been used to better understand the effect genetic risk mechanisms have on biological systems and clinical symptoms (e.g., Alloza et al., 2017). A recent study from our group has shown the importance of studying genetically at-risk, but clinically unaffected individuals, when investigating telomere length differences (Powell et al., 2017a). We previously found that unaffected first-degree relatives of $\mathrm{BD}$ patients have shorter telomeres compared to control participants, implying an association between familial risk for $\mathrm{BD}$ and shorter telomeres. This effect was not clear amongst $\mathrm{BD}$ cases, potentially as a result of lithium use, which was associated with longer telomeres within BD patients. Subsequently, PRS allows us to draw similar comparisons in any given population, with genetic risk being quantified empirically using genome-wide genotype data, as opposed to familial relatedness.

In addition to lithium use, other more commonly used pharmacotherapies have been implicated in affecting telomere length (Lindqvist et al., 2015; Monroy-Jaramillo et al., 2017). Recent studies have revealed that only depressed patients taking antidepressants have significantly shorter telomere lengths relative to controls; with the reports surmising that the effect is likely due to the more severe nature of depression in those requiring medication (Lindqvist et al., 2015; Needham et al., 2015). However, epidemiological studies independently reveal associations between antidepressant use and an increased risk for aging-related disease, which is irrespective of depression diagnosis (Hippisley-Cox et al., 2001; Gareri et al., 2002; Caughey et al., 2010). Thus, there is warrant for further investigation on the effects of antidepressant medication on telomere length, especially when used outside the context of depression, as this would allow one to tease apart the impact of psychiatric diagnosis from the impacts of medication.

Our study comprised of 351 participants from a South East London, United Kingdom population cohort. We aimed to investigate whether: (i) PRS for MDD, BD, or SCZ predicts shorter relative telomere length in the non-clinical majority of the sample; (ii) antidepressants impact on telomere length, 
irrespective of depression diagnosis, and (iii) if any significant associations from (i) or (ii) are additionally associated with risk for aging-related disease.

\section{MATERIALS AND METHODS}

\section{Participants}

A cohort study design was used to address the research questions. A total of 351 participants including 167 males (mean age of 50 (16.6 S.D.)) and 184 females (mean age of 47 (14.1 S.D.)) had their blood samples collected for DNA extraction as part of the South East London Community Health study (SELCoH; Hatch et al., 2011, 2016), see Table 1. Depression was the only common clinical psychiatric diagnosis in SELCoH, based on self-report data $(n=61)$. Current depression severity (at the time of blood collection) was coded as an ordinal measure $(0=$ no depression symptoms, 1 = mild depression symptoms, 2 = moderate-severe depression symptoms), using the Clinical Interview ScheduleRevised (CIS-R; Lewis et al., 1992), which uses an algorithm to approximate ICD-10 diagnoses (World Health Organisation [WHO], 1993).

To examine the relationship between PRS and telomere length, independent from the confounders of disease factors and medication in SELCoH, we split our sample into those without a reported depression diagnosis (dep-), and those with a reported depression diagnosis (dep+). DNA from participants was extracted from blood samples and this was used to calculate RTL and assay common genetic variation.

\section{Ethics Statement}

The SELCoH study received approval from King's College London research ethics committee, reference PNM/12/13-152.

TABLE 1 | The characteristics of our sample at the time of blood collection, including gender, age, BMI, smoking status, current antidepressant use and average polygenic risk scores (PRS), in depressed cases (Dep+) and non-depressed controls (Dep-),

\begin{tabular}{|c|c|c|}
\hline & Dep-sample & Dep+ sample \\
\hline$n$ & 290 & 61 \\
\hline Age & $48.43(15.58)$ & 48.36 (14.39) \\
\hline Sex (\% male) & $142(49)$ & $25(41)$ \\
\hline BMI & $27.04(5.39)$ & $27.99(6.25)$ \\
\hline \multirow[t]{6}{*}{ Ethnicity } & White British: 165 & White British: 35 \\
\hline & Black Caribbean: 24 & Black Caribbean: 6 \\
\hline & Black African: 33 & Black African: 3 \\
\hline & White other: 39 & White other: 12 \\
\hline & Non-white other: 18 & Non-white other: 4 \\
\hline & Mixed: 11 & Mixed: 1 \\
\hline \multirow[t]{3}{*}{ Smoking (n) } & Never: 122 & Never: 14 \\
\hline & Current: 53 & Current: 24 \\
\hline & Ex-smoker: 115 & Ex-smoker: 23 \\
\hline Currently taking antidepressants (n) & 10 & 30 \\
\hline PRS MDD & -0.0029 & 0.0137 \\
\hline PRS BD & -0.0141 & 0.067 \\
\hline PRS SCZ & -0.0164 & 0.078 \\
\hline
\end{tabular}

Informed written consent was obtained from all participants at the time of sample collection.

\section{Data Availability Statement}

Due to ethical restrictions SELCoH data is not publically available. Details on the SELCoH sample and requests to access phenotype data can be made here: http://www.slam. nhs.uk/research/selcoh/selcoh-projects. Access to genetic data requires local approval via the NIHR Bioresource (contact: bioresource@kcl.ac.uk).

\section{Aging-Related Disease}

We constructed an ordinal measure for aging-related disease, whereby 0 indicated no reported aging related disease, 1 indicated one reported aging-related disease, and 2 indicated two or more aging related diseases. Aging-related diseases included: type2 diabetes, arthritis, cardiovascular disease, stroke, high blood pressure, and cancer.

\section{DNA Extraction and Telomere Assessment}

$10 \mathrm{~mL}$ of blood was collected from participants in tubes containing EDTA (BD Vacutainer; BD, NJ, United States) and stored at $-80^{\circ} \mathrm{C}$. DNA was then extracted using a standard inhouse protocol (Freeman et al., 2003) and stored at $-80^{\circ} \mathrm{C}$. All samples had 260/280 ratios of between 1.7 and 1.9, tested using the Nanodrop D1000 (Thermoscientific, Wilmington, DE, United States).

To assess relative telomere length (RTL), we performed a modified version of a quantitative polymerase reaction (qPCR) protocol by Cawthon (2009), as previously described (Powell et al., 2017a; Vincent et al., 2017). The protocol involves two separate qPCRs performed on separate 384-well plates with DNA samples pipetted into identical wells on each plate. In the first reaction, we assayed the telomere repeat region (TTAGGG). In the second reaction, we assayed a single copy gene, albumin, which we used as an internal control to correct for differences in DNA concentration between samples (Cawthon, 2009). The telomere/albumin ratio was used to calculate RTL.

On each plate, six negative controls consisting of RNase-free water were used to screen for any DNA contamination. An eight-point dilution series using human leukocyte genomic DNA $(0.47,0.94,1.88,3.75,7.5,15,30$, and $60 \mathrm{ng})$ was used on each plate to allow for absolute quantification of each sample and to account for any differences in efficiency between the telomere and albumin reactions. All reactions were performed using three technical replicates. Each qPCR mix for the telomere reactions consisted of $10 \mu \mathrm{L}$ of $2 \mathrm{x}$ qPCR Mastermix with SYBR Green (Primer Design, Southampton, United Kingdom), $5 \mu \mathrm{L}$ or RNase free water, $12 \mathrm{ng}$ of DNA, $1000 \mathrm{nM}$ of telg, 5'-ACACTAAGGTTTGGGTTTGGGTTTGGGTTTGGGTTAG TGT-3' and 800nM of telc, 5' ${ }^{\prime}$-TGTTAGGTATCCCTATCCCT ATCCCTATCCCTATCCCTAACA-3'. Four stages made up the thermocycling conditions as follows: Stage $1: 95^{\circ} \mathrm{C}$ for $15 \mathrm{~min}$, Stage 2: 2 cycles for $15 \mathrm{~s}$ at $94^{\circ} \mathrm{C}$ and $49^{\circ} \mathrm{C}$, Stage 3: 25 cycles at 
$94^{\circ} \mathrm{C}$ for $15 \mathrm{~s}, 10 \mathrm{~s}$ at $62^{\circ} \mathrm{C}$, and $15 \mathrm{~s}$ at $73^{\circ} \mathrm{C}$ (data collection), Stage 4: dissociation curve (primer specificity detection).

The same reagents and quantities were used for the albumin reactions, apart from the albumin forward and reverse primers replaced the telomere primers. Quantities of the albumin forward and reverse primers were adjusted to $765 \mathrm{nM}$ for the forward primer albu, 5'-CGGCGGCGGGCGGCGCGGGCTGGGCGGA AATGCTGCACAGAATCCTT-3' and $930 \mathrm{nM}$ for the reverse primer albd, 5'-GCCCGGCCCGCCGCGCCCGTCCCGCCGG AAAAGCATGGTCGCCTGTT- $3^{\prime}$. The thermocycling conditions for the albumin reaction consisted of four stages: Stage $1: 95^{\circ} \mathrm{C}$ for $15 \mathrm{~min}$, Stage 2: 2 cycles for $15 \mathrm{~s}$ at $94^{\circ} \mathrm{C}$ and $49^{\circ} \mathrm{C}$, Stage 3: 33 cycles at $94^{\circ} \mathrm{C}$ for $15 \mathrm{~s}, 10 \mathrm{~s}$ at $62^{\circ} \mathrm{C}$, and $15 \mathrm{~s}$ at $88^{\circ} \mathrm{C}$ (data collection), Stage 4: dissociation curve (primer specificity detection).

Reactions were performed using either the ABI Prism 7900HT Sequence Detection System (Thermofisher Scientific, MA, United States) or the QuantStudio 7 Flex Real-Time PCR System (Thermofisher Scientific).

\section{Genotyping and Quality Control (Target Dataset)}

DNA samples were sent to the Affymetrix Research Services Laboratory in Santa Clara, California, CA, United States. Genotyping for SELCoH was assayed using the United Kingdom Biobank Axiom Array (r3) which comprises of 820,967 genetic markers (Affymetrix, California, CA, United States). Genotype data was put through quality control measures as outlined by Coleman et al. (2016), using PLINK v1.07 (Purcell et al., 2007) and mapped to genomic build hg19. Specifically, patient samples were excluded if there was greater than $5 \%$ missingness in genotype data, and individual SNPs were excluded if there was greater than 5\% missingness. A minor allele frequency (MAF) threshold was set to 0.05 , and a Hardy-Weinberg threshold of 0.00001 , in keeping with what's recommended for smaller sample sizes (Coleman et al., 2016).

The absence of sample mismatching was confirmed using sex checks, where genetic sex was compared to phenotypic sex. The genome-wide Identity by Descent (IBD) analysis which is performed between pairs of samples, measured the probable number of shared alleles at any given marker, and was used to identify and exclude relatives within our sample. Relatives were identified as those with a PI-HAT (proportion of IBD) threshold of greater than 0.1875 ; where 0.5 represents firstdegree relatives and 0.25 represents second-degree relatives. Only a single member from each family were retained post quality control. Following quality control, the sample consisted of 351 unrelated individuals, for which we had both genome-wide genotype data and telomere length data.

\section{Polygenic Risk Score Quantification PRSice Software}

Individualized Polygenic Risk Scores within our sample were calculated using PRSice, a PRS quantification software (Euesden et al., 2015). The software uses summary results from previously performed, well-powered GWAS (the base dataset) to generate
PRSs in our sample, SELCoH (the target dataset). Briefly, PRSice works by first clumping SNPs in the genotype PLINK files corresponding to the target dataset and removing those in high linkage disequilibrium, as this can falsely inflate polygenic scores. Subsequently, within the target dataset the number of risk alleles at a particular SNP is multiplied by that SNP's effect size (established in the base dataset), and then all the SNP information is summed. The user can define which SNPs to include in the PRS. For all analyses we set a $p$-value threshold of $p=0.1$, whereby we included all SNPs under this threshold from our base datasets to calculate polygenic risk scores in our target dataset.

\section{Base Datasets}

The MDD base dataset (summary statistics) was obtained from the Psychiatric Genomics Consortium (PGC) and represents the largest GWAS for depression to-date, consisting of 135,458 MDD cases and 344,901 controls (Wray et al., 2018). The base dataset for BD consists of GWAS results for 7,481 cases and 9,250 controls (Sklar et al., 2011). For the BD GWAS, SNP positions were lifted over from hg18 to hg19 build using UCSC LiftOver tool (Kuhn et al., 2013). The base dataset for SCZ consists of insights from a multi-stage schizophrenia genomewide association study of up to 36,989 cases and 113,075 controls (Ripke et al., 2014). All base datasets were downloaded from the PGC website ${ }^{1}$.

\section{Population Covariates}

To reduce noise in our analyses as a result of ancestry differences within the sample, we generated population covariates (PCs) using multidimensional scaling in PLINK, via the PRSice software, which allowed us to detect and adjust for population structure in our analyses (Patterson et al., 2006; Price et al., 2006). Population covariates were incrementally tested for association via scatter charts (e.g., PC1 vs. PS2, PC2 vs. PC3, PC3 vs. PC4, etc.) until a normal distribution was achieved. A normal distribution was achieved after the first seven PCs, and thus this is what we used in our downstream analyses.

\section{Statistical Analysis \\ RTL Calculation}

A standard deviation of less than 0.5 was required for at least two of the three cycle threshold $\left(\mathrm{C}_{t}\right)$ technical triplicates for a sample to be included in downstream analysis. $C_{q}$ values were then created from the remaining $C_{t}$ values by relating them to absolute quantities as part of a standard curve. RTL was then calculated by dividing each sample's mean $C_{q}$ value from the telomere reaction by each sample's mean $C_{q}$ value from the albumin reaction. RTL was then logtransformed to allow for parametric analysis. Outliers were identified as those data points greater than two standard deviations from the mean and subsequently removed. As a final check, we performed a one-tailed Pearson correlation test to confirm there was a negative correlation between $\log (\mathrm{RTL})$ and age.

${ }^{1}$ http://www.med.unc.edu/pgc/results-and-downloads/results 


\section{PRS and its Relationship to Telomere Length (Dep-Sample Only)}

To determine the effect of each PRS on telomere length we performed three independent linear regressions, whereby $\log (\mathrm{RTL})$ was selected as our outcome; age, BMI, PCs 1-7, plate batch, smoking status (former, current, never), gender, and ethnicity were selected as covariates; and PRS was selected as our independent variable.

\section{Antidepressant Use and Telomere Length (Full Sample)}

The relationship between antidepressant use and telomere length was investigated using a linear regression, whereby log-telomere length was selected as the outcome variable; age, BMI, PCs 17, plate batch, smoking status (former, current, never), gender, ethnicity, depression diagnosis were included as covariates; and antidepressant use was used as our independent variable.

\section{Antidepressant Use and Aging-Related Disease (Full Sample)}

An ordinal logistic regression was used to determine the relationship between antidepressant use and the number of aging-related diseases (0/1/2). Number of aging-related diseases was selected as our outcome variable; age, BMI, PCs 1-7, smoking status (former, current, never), gender, ethnicity, and lifetime depression diagnosis were selected as covariates; with antidepressant use selected as the independent variable.

\section{Sensitivity Analyses}

We performed a series of sensitivity analyses to determine the relationship between telomere length and physical illnesses/medication use, and the potential confounding effects of depression severity.

\section{Power Calculation}

Power calculations indicate we have $100 \%$ power to detect smallmoderate effect sizes (effect size $=0.3$ ) for analyses (i) to (iii), given our sample size, with an $\alpha=0.05$.

\section{RESULTS}

\section{Quality Control Checks}

Standard curves from all reactions showed an $R^{2} \geq 0.98$ between quantity of known DNA and $C_{t}$ values. Negative controls showed no amplification on any of the plates and a single peak was detected for the dissociation curves (melting curves) across all plates, demonstrating that binding specificity of the primers to the DNA was achieved to a high degree, see Supplementary Information. The telomere reaction achieved a mean efficiency of $90 \%$ and the albumin reaction achieved a mean efficiency of $79 \%$. Efficiencies were all corrected via a standard curve on each of the plates and all samples which didn't pass our quality control criteria were removed from any further analyses (17 samples).

\section{Telomere Length and Chronological Age}

A one-tailed Pearson's correlation showed that relative telomere length (adjusted for inter-plate variability) is negatively correlated

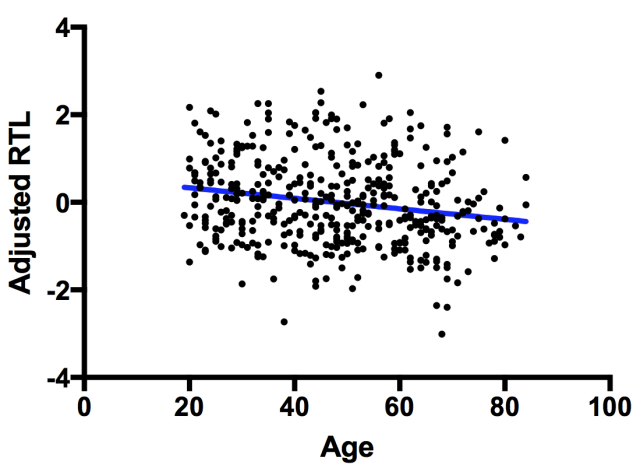

FIGURE 1 | Correlation between telomere length and chronological age. A scatterplot showing a significant negative correlation between chronological age ( $x$-axis) and adjusted RTL ( $y$-axis). The blue line represents a line of best fit.

with age in our whole sample, $r(351)=-0.223, \mathrm{p} 1.20 \mathrm{E}-05$ (Figure 1) as expected.

\section{Polygenic Risk for Psychiatric Disorders and Telomere Length}

The regression model examining the effect of polygenic risk for SCZ on $\log (\mathrm{RTL})$ did not reveal a significant association $(F(1,265)=1.622, p=0.204)$. Similarly, we did not find a significant association between the polygenic risk for $\mathrm{BD}$ and $\log (\mathrm{RTL} ; F(1,265)=1.872, P=0.172)$, nor between polygenic risk for MDD and $\log (\mathrm{RTL} ; F(1,265)=0.519, P=0.472)$, see Figure 2 .

\section{The Effect of Antidepressants Use on Telomere Length}

Antidepressant use was significantly associated with telomere length in our total sample, which was irrespective of depression diagnosis $(F(1,325)=6.575, P=0.011$, variance explained $=2 \%)$, see Figure 3.

\section{Antidepressant Use and Aging Related Disease}

We found that those currently taking antidepressants also had a higher frequency of aging-related disease, relative to those not currently taking an antidepressant. (Estimate $=-0.981(95 \%$ C.I. $=-1.878,-0.084), p=0.032$ ), see Figure 4.

\section{Sensitivity Analyses}

Telomere length and Aging-Related Disease

We performed ordinal logistic regressions to determine if $\log (\mathrm{RTL})$ predicts number of aging-related diseases $(0,1,2)$ whilst covarying for age, BMI, PCs 1-7, smoking status (former, current, never), gender, ethnicity, lifetime depression diagnosis and antidepressant use. $\log (\mathrm{RTL})$ did not predict number of ageing-related diseases $(p>0.05)$.

\section{Telomere Length, Medication Use and Disease}

We performed linear regressions which included age, sex, ethnicity and BMI as covariates, and $\log (\mathrm{RTL})$ as the outcome 

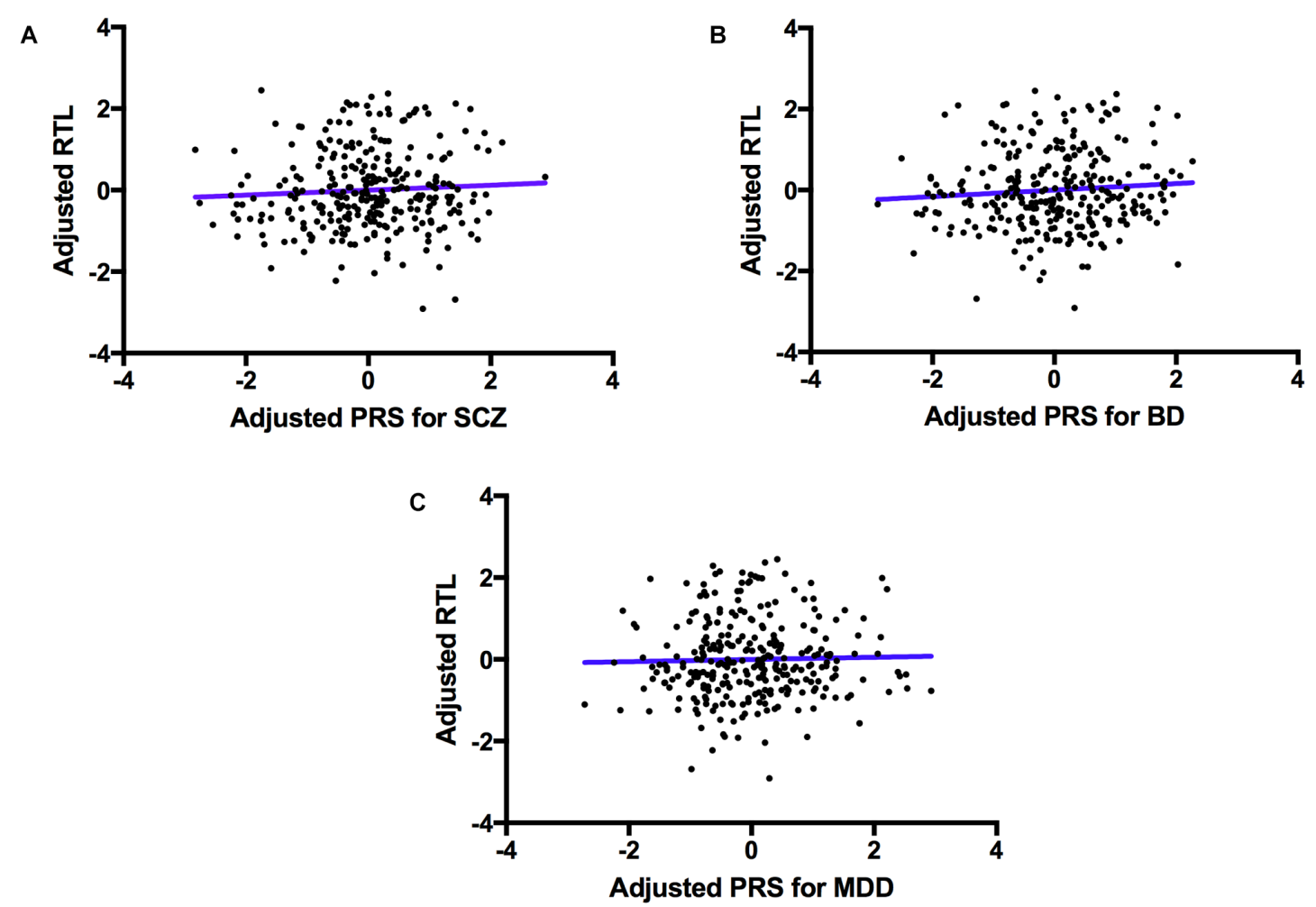

FIGURE 2 | The association between polygenic risk for psychiatric disorders and telomere length. A scatter plot showing PRSs for SCZ (A, top left), BD (B, top right) and $\operatorname{MDD}(\mathbf{C}$, bottom) adjusted for PCs 1-7 (x-axis), and log(RTL) adjusted for age, sex, ethnicity, BMl, smoking status and telomere plate batch ( $y$-axis). The blue line represents the line of best fit.

variable, alongside self-reported disease or medication use. No diseases or medications predicted $\log (\mathrm{RTL}), p>0.05$, see Supplementary Information for further details.

\section{Telomere Length, Antidepressant Use and Aging-Related Disease}

We further tested whether telomere length mediated the association between antidepressant use and risk for aging-related disease. So we repeated analysis (iv) as described above: An ordinal logistic regression was used to determine the relationship between antidepressant use and the number of aging-related diseases ( $0 / 1 / 2+$; outcome variable). However, we also included $\log (\mathrm{RTL})$ as a covariate. The relationship between antidepressant use and number of aging-related diseases remained significant $(p<0.05)$, suggesting the effect was not mediated by telomere length.

\section{Depression Severity at Blood Collection, and Telomere Length}

To confirm depression severity did not confound analyses investigating the effects of antidepressants on $\log (\mathrm{RTL})$, we repeated analysis (iii), however, we further included depression severity ( $0=$ none, $1=$ mild, $2=$ moderate/severe $)$ as a covariate, and found that antidepressant use still predicted $\log$ (RTL; $p<0.05$ ), suggesting episode severity was not confounding our result.

\section{DISCUSSION}

Previous studies have revealed higher rates of aging-related diseases amongst psychiatric disorder patients, with some studies indicating that shorter telomere length (and faster aging) may be the cause (Hippisley-Cox et al., 2001; Gareri et al., 2002; Caughey et al., 2010). The first aim of our study was to clarify whether genetic risk for psychiatric disorders also carries risk for shorter telomere length. To achieve this aim we generated PRS for MDD, BD, and SCZ, and assessed the relationship between these PRS and telomere length measurements in a cohort of individuals with no history of psychiatric health problems. We found no evidence to suggest that genetic risk for psychiatric disorders also contributes to telomere shortening, Figure 2. In terms of translational medicine, our results suggest that although polygenic risk scoring may be useful in predicting those at risk for psychiatric disorders, current psychiatric polygenic risk scores alone may not be useful in predicting those who are also susceptible to shorter telomeres and aging-related diseases. Instead, our results support previous work indicating the importance of environmental factors associated with psychiatric diagnosis, in accelerating telomere shortening (Kessler et al., 2005; Whiteford et al., 2013).

Indeed, we found evidence that antidepressant use was associated with shorter telomere length, an effect which was independent of depression diagnosis. This partially corroborates 


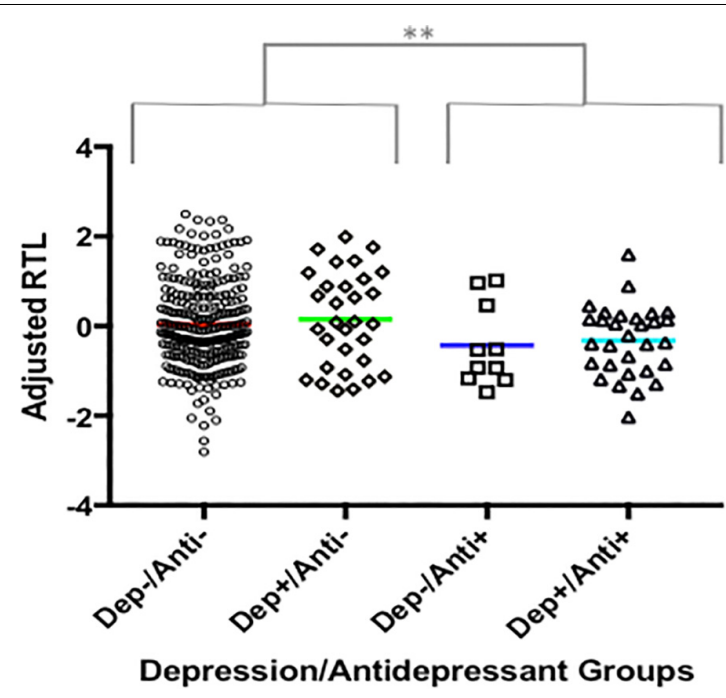

FIGURE 3 | The association between antidepressant use and RTL. A plot showing adjusted RTL in participants: (i) without depression and who are not currently taking antidepressants (Dep-/Anti-), (ii) with a depression diagnosis and who are not currently taking antidepressants (Dep+/Anti-), (iii) without depression who are currently taking antidepressants, (iv) with depression who are currently taking antidepressants. Participants currently taking antidepressants had significantly shorter RTL compared to those not taking antidepressants, irrespective of depression diagnosis. The symbol

'**' indicates a difference of $p \leq 0.01$.

previous reports which found that only depressed patients currently taking antidepressants have shorter telomeres (Lindqvist et al., 2015; Needham et al., 2015). However, in contrast to previous studies which have suggested that antidepressant use is a proxy for current depression severity, and that this is what drives the association with shorter telomeres, our results show that even non-depressed participants who are taking antidepressants for other purposes (e.g., sleep) had similarly short telomere lengths, Figure 3. We further confirmed that current depression severity did not differ between depressed patients who were and were not taking antidepressants at the time of blood collection, suggesting the effect was not driven by current depression severity. One possible explanation is that antidepressants are increasing the proliferation of blood cells in users, and the knock-on effect is telomere shortening. Indeed, research using lymphoblastoid cell lines (resembling white blood cells), hippocampal progenitor cell lines, and in vivo studies of the hippocampus, support the notion that antidepressants increase proliferation (Manev et al., 2001; Breitfeld et al., 2017; Powell et al., 2017b). However, some work indicates that antidepressants may additionally increase the activity of telomerase, which is an enzyme involved in telomere maintenance and elongation (Bersani et al., 2015), therefore more work is needed to better understand the effects of antidepressants on telomere length and proliferation over time.

Interestingly, we additionally found an association between antidepressant use and risk for aging-related disease; with antidepressant use predicting a higher number of aging-related diseases, an effect which was independent of depression case/control status, Figure 4. This matches recent epidemiological reports that antidepressant use is associated with an increased risk for aging-related disorders such as cardiovascular disease (Musselman et al., 1998; Lichtman et al., 2008; Hamer et al., 2011). Interestingly, our sensitivity analyses suggest that the relationship between antidepressant use and aging-related disease is not mediated by telomere length variability, such that antidepressant use is independently associated with both risk for aging-related disease and shorter telomere length. These results indicate one of two things. First, that antidepressants increase telomere shortening and risk for aging-related disease via independent mechanisms. Second, and more likely, that antidepressant use, or prescription, is more common amongst those who suffer from depression (or related conditions such as sleep problems), and who also suffer

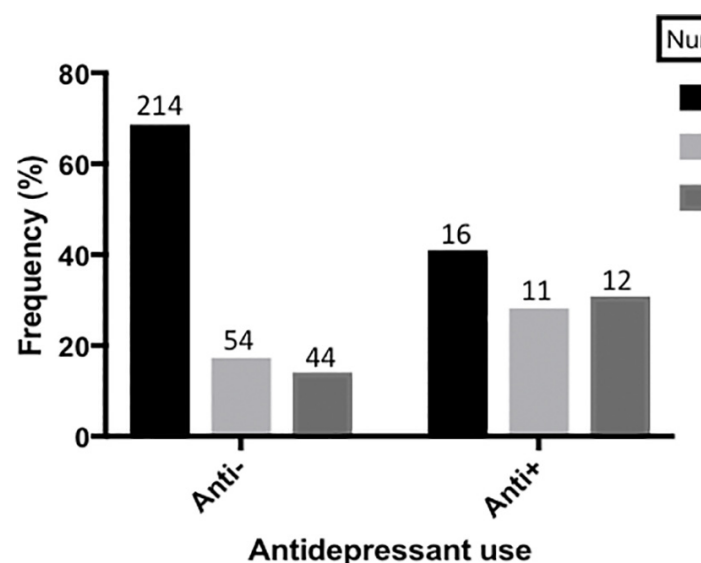

Number of ageing-related diseases

0

1

2

Antidepressant use

FIGURE 4 | Antidepressant use and aging-related disease. A bar chart showing the frequency of participants with 0, 1 or 2(+) aging-related diseases, split by those who are not currently taking antidepressants and those who are. Actual sample size within each group is shown on top of each bar. There is a significant difference in the frequency of aging-related disease between the two groups $(p<0.05)$. Aging-related diseases included: type-2 diabetes, arthritis, cardiovascular disease, stroke, high blood pressure, and cancer. 
from chronic debilitating aging-related disease. Nevertheless, the combination of previously reported epidemiological data linking antidepressant use with aging-related disease, and molecular data reported here, warrants further consideration of the long-term impact of antidepressant use on aging-related phenotypes. In particular, studies recruiting antidepressant users (both depressed and non-depressed) as part of a longitudinal design may help in discerning causality.

The main limitations of our study are the small number of individuals taking antidepressants, and the fact we may be underpowered to detect small effect sizes. Nevertheless, our study is the first to suggest that: (i) genetic risk for psychiatric disorders does not predict faster biological aging, (ii) antidepressant use is associated with shorter telomeres independently of depression diagnosis, (iii) antidepressant use is associated with an increased number of aging-related diseases, independently of depression diagnosis. Our work suggests that the relationship between antidepressant use and risk for aging-related disease may need to be reconsidered.

\section{CONCLUSION}

We found no evidence to suggest that genetic risk for psychiatric disorders also contribute to faster telomere shortening, highlighting the potential importance of environmental factors in mediating physical disease comorbidity. We did, however, find an association between antidepressant use and telomere length, with antidepressant use being associated with shorter telomere length. In addition, we found antidepressant use to be associated with a higher number of aging-related diseases in participants, replicating previous epidemiological evidence. Further work is now needed to test whether antidepressants induce telomere shortening via their proliferative effects, and how antidepressant use relates to aging-related disease.

\section{AUTHOR CONTRIBUTIONS}

GB, LG, SF, SH, and MH did the sample collection, interpretation of results, final approval for publication. CL and ST interpreted

\section{REFERENCES}

Alloza, C., Bastin, M. E., Cox, S. R., Gibson, J., Duff, B., Semple, S. I., et al. (2017). Central and non-central networks, cognition, clinical symptoms, and polygenic risk scores in schizophrenia. Hum Brain Mapp. 38, 5919-5930. doi: 10.1002/hbm.23798

Bersani, F. S., Lindqvist, D., Mellon, S. H., Penninx, B. W., Verhoeven, J. E., Révész, D., et al. (2015). Telomerase activation as a possible mechanism of action for psychopharmacological interventions. Drug Discov. Today 20, 13051309. doi: 10.1016/j.drudis.2015.06.016

Blackburn, E. H. (2001). Switching and signaling at the telomere. Cell 106, 661-673. doi: 10.1016/S0092-8674(01)00492-5

Blasco, M. A. (2005). Telomeres and human disease: ageing, cancer and beyond. Nat. Rev. Genet. 6, 611-622. doi: 10.1038/nrg1656

Breitfeld, J., Scholl, C., Steffans, M., Laje, G., and Stingl, J. C. (2017). Gene expression and proliferation biomarkers for antidepressant treatment resistance. Transl. Psychiatry 7:e1061. doi: 10.1038/tp.2017.16 the results and provided the final approval for publication. AP and TP created and designed the work, acquired the data, analyzed and interpreted the data, prepared and revised the manuscript, and provided the final approval for publication.

\section{FUNDING}

AP is funded by a Rayne Foundation Ph.D. studentship (TRT - M14717) and TP is funded by a Medical Research Council Skills Development Fellowship (MR/N014863/1). The current project was funded by a Psychiatry Research Trust Grant (92 Branthwaite) awarded to TP and GB. SELCoH was supported by the Biomedical Research Nucleus data management and informatics facility at South London and Maudsley NHS Foundation Trust, which is funded by the National Institute for Health Research (NIHR) Mental Health Biomedical Research Center at South London and Maudsley NHS Foundation Trust and King's College London and a joint infrastructure grant from Guy's and St Thomas' Charity and the Maudsley Charity. Phase 3 of the SELCoH study was also funded by the Maudsley Charity. MH, SH, SF, LG, $\mathrm{GB}$, and $\mathrm{CL}$ are supported by the National Institute for Health Research (NIHR) Mental Health Biomedical Research Center, South London and Maudsley NHS Foundation Trust and King's College London. The views expressed are those of the authors and not necessarily those of the NHS, the NIHR or the Department of Health and Social Care. The funding sources had no role in the study the design, in the collection, analysis, and interpretation of data, in the writing of the report and in the decision to submit the article for publication.

\section{SUPPLEMENTARY MATERIAL}

The Supplementary Material for this article can be found online at: https://www.frontiersin.org/articles/10.3389/fgene. 2018.00468/full\#supplementary-material

Caughey, G. E., Roughead, E. E., Shakib, S., McDermott, R. A., Vitry, A. I., and Gilbert, A. L. (2010). Comorbidity of chronic disease and potential treatment conflicts in older people dispensed antidepressants. Age Ageing 39, 488-494. doi: 10.1093/ageing/afq055

Cawthon, R. M. (2009). Telomere length measurement by a novel monochrome multiplex quantitative PCR method. Nucleic Acids Res. 37:e21. doi: 10.1093/nar/ gkn1027

Chang, C.-K., Hayes, R. D., Perera, G., Broadbent, M. T. M., Fernandes, A. C., Lee, W. E., et al. (2011). Life expectancy at birth for people with serious mental illness and other major disorders from a secondary mental health care case register in London. PLoS One 6:e19590. doi: 10.1371/journal.pone.00 19590

Chwastiak, L. A., Rosenheck, R. A., McEvoy, J. P., Keefe, R. S., Swartz, M. S., and Lieberman, J. A. (2006). Special section on catie baseline data: interrelationships of psychiatric symptom severity, medical comorbidity, and functioning in schizophrenia. Psychiatr. Serv. 57, 1102-1109. doi: 10.1176/ps.2006.57.8. 1102 
Clarke, T.-K., Lupton, M. K., Fernandez-Pujals, A. M., Starr, J., Davies, G., Cox, et al. (2016). Common polygenic risk for autism spectrum disorder (ASD) is associated with cognitive ability in the general population. Mol. Psychiatry 21, 419-425. doi: 10.1038/mp.2015.12

Coleman, J. R. I., Euesden, J., Patel, H., Folarin, A. A., Newhouse, S., and Breen, G. (2016). Quality control, imputation and analysis of genome-wide genotyping data from the Illumina HumanCoreExome microarray. Brief. Funct. Genomics 15, 298-304. doi: 10.1093/bfgp/elv037

Collins, K., and Mitchell, J. R. (2002). Telomerase in the human organism. Oncogene 21, 564-579. doi: 10.1038/sj.onc.1205083

Davies, S. C. (2014). Annual Report of the Chief Medical Officer. Annual Report of the Chief Medical Officer. Available at: https://www.gov.uk/government/ organisations/department-of-health

de Lange, T. (2002). Protection of mammalian telomeres. Oncogene 21, 532-540. doi: 10.1038/sj.onc. 1205080

Elvsåshagen, T., Vera, E., Bøen, E., Bratlie, J., Andreassen, O. A., Josefsen, D., et al. (2011). The load of short telomeres is increased and associated with lifetime number of depressive episodes in bipolar II disorder. J. Affect. Disord. 135, 43-50. doi: 10.1016/j.jad.2011.08.006

Euesden, J., Lewis, C. M., and O’Reilly, P. F. (2015). PRSice: polygenic risk score software. Bioinformatics 31, 1466-1468. doi: 10.1093/bioinformatics/btu848

Forty, L., Ulanova, A., Jones, L., Jones, I., Gordon-Smith, K., Fraser, C., et al. (2014). Comorbid medical illness in bipolar disorder. Br. J. Psychiatry 205, 465-472. doi: 10.1192/bjp.bp.114.152249

Freeman, B., Smith, N., Curtis, C., Huckett, L., Mill, J., and Craig, I. W. (2003). DNA from buccal swabs recruited by mail: evaluation of storage effects on long-term stability and suitability for multiplex polymerase chain reaction genotyping. Behav. Genet. 33, 67-72. doi: 10.1023/A:10210556 17738

Gareri, P., De Fazio, P., and De Sarro, G. (2002). Neuropharmacology of depression in aging and age-related diseases. Ageing Res. Rev. 1, 113-134. doi: 10.1016/ S0047-6374(01)00370-0

Hamer, M., Batty, G. D., Seldenrijk, A., and Kivimaki, M. (2011). Antidepressant medication use and future risk of cardiovascular disease: the Scottish health survey. Eur. Heart J. 32, 437-442. doi: 10.1093/eurheartj/ ehq438

Hartmann, N., Boehner, M., Groenen, F., and Kalb, R. (2010). Telomere length of patients with major depression is shortened but independent from therapy and severity of the disease. Depress. Anxiety 27, 1111-1116. doi: 10.1002/da. 20749

Hatch, S. L., Frissa, S., Verdecchia, M., Stewart, R., Fear, N. T., Reichenberg, A., et al. (2011). Identifying socio-demographic and socioeconomic determinants of health inequalities in a diverse London community: the South East London Community Health (SELCoH) study. BMC Public Health 11:861. doi: 10.1186/ 1471-2458-11-861

Hatch, S. L., Gazard, B., Williams, D. R., Frissa, S., Goodwin, L., SELCoH Study Team, et al. (2016). Discrimination and common mental disorder among migrant and ethnic groups: findings from a South East London Community sample. Soc. Psychiatry Psychiatr. Epidemiol. 51, 689-701. doi: 10.1007/s00127016-1191-x

Hippisley-Cox, J., Pringle, M., Hammersley, V., Crown, N., Wynn, A., Meal, A., et al. (2001). Antidepressants as risk factor for ischaemic heart disease: casecontrol study in primary care. BMJ 323, 666-669. doi: $10.1136 / \mathrm{bmj} .323 .73$ 14.666

Kang, H.-J., Kim, S.-Y., Bae, K.-Y., Kim, S.-W., Shin, I.-S., Yoon, J.-S., et al. (2015). Comorbidity of depression with physical disorders: research and clinical implications. Chonnam. Med. J. 51, 8-18. doi: 10.4068/cmj.2015.51.1.8

Kao, H.-T., Cawthon, R. M., DeLisi, L. E., Bertisch, H. C., Ji, F., Gordon, D., et al. (2008). Rapid telomere erosion in schizophrenia. Mol. Psychiatry 13, 118-119. doi: 10.1038/sj.mp.4002105

Kemp, D. E., Gao, K., Chan, P., Ganocy, S. J., Findling, R. L., and Calabrese, J. R. (2010). Medical comorbidity in bipolar disorder: relationship between illnesses of the endocrine/metabolic system and treatment outcome. Bipolar Disord. 12, 404-413. doi: 10.1111/j.1399-5618.2010.00823.x

Kessler, R. C., Chiu, W. T., Demler, O., and Walters, E. E. (2005). Prevalence, severity, and comorbidity of 12-month DSM-IV disorders in the national comorbidity survey replication. Arch. Gen. Psychiatry 62, 617-627. doi: 10.1001/ archpsyc.62.6.617
Kuhn, R. M., Haussler, D., and Kent, W. J. (2013). The UCSC genome browser and associated tools. Brief. Bioinform. 14, 144-161. doi: 10.1093/bib/ bbs038

Lewis, G., Pelosi, A. J., Araya, R., and Dunn, G. (1992). ). Measuring psychiatric disorder in the community: a standardized assessment for use by lay interviewers. Psychol. Med. 22, 465-486. doi: 10.1017/S003329170003 0415

Lichtman, J. H., Bigger, J. T., Blumenthal, J. A., Frasure-Smith, N., Kaufmann, P. G., Lespérance, F., et al. (2008). Depression and coronary heart disease: recommendations for screening, referral, and treatment: a science advisory from the American Heart Association Prevention Committee of the Council on Cardiovascular Nursing, Council on Clinical Cardiology, Council on Epidemiology and Prevention, and Interdisciplinary Council on Quality of Care and Outcomes Research: endorsed by the American Psychiatric Association. Circulation 118, 1768-1775. doi: 10.1161/CIRCULATIONAHA.108.19 0769

Lima, I. M. M., Barros, A., Rosa, D. V., Albuquerque, M., Malloy-Diniz, L., Neves, F. S., et al. (2015). Analysis of telomere attrition in bipolar disorder. J. Affect. Disord. 172, 43-47. doi: 10.1016/j.jad.2014.09.043

Lindqvist, D., Epel, E. S., Mellon, S. H., Penninx, B. W., Révész, D., Verhoeven, J. E., et al. (2015). Psychiatric disorders and leukocyte telomere length: underlying mechanisms linking mental illness with cellular aging. Neurosci. Biobehav. Rev. 55, 333-364. doi: 10.1016/j.neubiorev.2015.05.007

Lung, F.-W., Chen, N. C., and Shu, B.-C. (2007). Genetic pathway of major depressive disorder in shortening telomeric length. Psychiatr. Genet. 17, 195-199. doi: 10.1097/YPG.0b013e32808374f6

Mamdani, F., Rollins, B., Morgan, L., Myers, R. M., Barchas, J. D., Schatzberg, A. F., et al. (2015). Variable telomere length across post-mortem human brain regions and specific reduction in the hippocampus of major depressive disorder. Transl. Psychiatry 5:e636. doi: 10.1038/tp.2015.134

Manev, H., Uz, T., Smalheiser, N. R., and Manev, R. (2001). Antidepressants alter cell proliferation in the adult brain in vivo and in neural cultures in vitro. Eur. J. Pharmacol. 411, 67-70. doi: 10.1016/S0014-2999(00)00904-3

Meeuwisse-Pasterkamp, S. H., van der Klauw, M. M., and Wolffenbuttel, B. H. (2008). Type 2 diabetes mellitus: prevention of macrovascular complications. Expert Rev. Cardiovasc. Ther. 6, 323-341. doi: 10.1586/14779072.6. 3.323

Menear, M., Doré, I., Cloutier, A.-M., Perrier, L., Roberge, P., Duhoux, A., et al. (2015). The influence of comorbid chronic physical conditions on depression recognition in primary care: a systematic review. J. Psychosom. Res. 78, 304-313. doi: 10.1016/j.jpsychores.2014.11.016

Monroy-Jaramillo, N., Dyukova, E., and Walss-Bass, C. (2017). Telomere length in psychiatric disorders: is it more than an ageing marker? World J. Biol. Psychiatry doi: 10.1080/15622975.2016.1273550 [Epub ahead of print].

Musselman, D. L., Evans, D. L., and Nemeroff, C. B. (1998). The relationship of depression to cardiovascular disease. Arch. Gen. Psychiatry 55, 580-592. doi: $10.1001 /$ archpsyc.55.7.580

Naylor, C., Parsonage, M., Mcdaid, D., Knapp, M., Fossey, M., and Galea, A. (2012). Long-Term Conditions and Mental Health The Cost of Co-morbidities. Available at: https://www.kingsfund.org.uk/sites/files/kf/field/field_publication_file/ long-term-conditions-mental-health-cost-comorbidities-naylor-feb12.pdf

Needham, B. L., Mezuk, B., Bareis, N., Lin, J., Blackburn, E. H., and Epel, E. S. (2015). Depression, anxiety and telomere length in young adults: evidence from the National Health and Nutrition Examination Survey. Mol. Psychiatry 20, 520-528. doi: 10.1038/mp.2014.89

Oh, H., Wang, S. C., Prahash, A., Sano, M., Moravec, C. S., Taffet, G. E., et al. (2003). Telomere attrition and Chk2 activation in human heart failure. Proc. Natl. Acad. Sci. U.S.A. 100, 5378-5383. doi: 10.1073/pnas.08360 98100

Patterson, N., Price, A. L., and Reich, D. (2006). Population structure and eigenanalysis. PLoS Genet. 2:e190. doi: 10.1371/journal.pgen.00 20190

Powell, T. R., Dima, D., Frangou, S., and Breen, G. (2017a). Telomere length and bipolar disorder. Neuropsychopharmacology 43, 445-453. doi: 10.1038/npp. 2017.125

Powell, T. R., Murphy, T., de Jong, S., Lee, S. H., Tansey, K. E., Hodgson, K., et al. (2017b). The genome-wide expression effects of escitalopram and its relationship to neurogenesis, hippocampal volume, and antidepressant 
response. Am. J. Med. Genet. B Neuropsychiatr. Genet. 174, 427-434. doi: 10. 1002/ajmg.b.32532

Price, A. L., Patterson, N. J., Plenge, R. M., Weinblatt, M. E., Shadick, N. A., and Reich, D. (2006). Principal components analysis corrects for stratification in genome-wide association studies. Nat. Genet. 38, 904-909. doi: 10.1038/ ng1847

Purcell, S., Neale, B., Todd-Brown, K., Thomas, L., Ferreira, M. A. R., Bender, D., et al. (2007). PLINK: a tool set for whole-genome association and populationbased linkage analyses. Am. J. Hum. Genet. 81, 559-575. doi: 10.1086/519795

Rai, D., Stansfeld, S., Weich, S., Stewart, R., Mcbride, O., Brugha, T., et al. (2014). Comorbidity in Mental and Physical Illness. Available at: http://content.digital. nhs.uk/catalogue/PUB21748/apms-2014-comorbidity.pdf

Ripke, S., Neale, B. M., Corvin, A., Walters, J. T. R., Farh, K.-H., Holmans, P. A., et al. (2014). Biological insights from 108 schizophrenia-associated genetic loci. Nature 511, 421-427. doi: 10.1038/nature13595

Samani, N. J., Boultby, R., Butler, R., Thompson, J. R., and Goodall, A. H. (2001). Telomere shortening in atherosclerosis. Lancet 358, 472-473. doi: 10.1016/ S0140-6736(01)05633-1

Simon, N. M., Smoller, J. W., McNamara, K. L., Maser, R. S., Zalta, A. K., Pollack, M. H., et al. (2006). telomere shortening and mood disorders: preliminary support for a chronic stress model of accelerated aging. Biol. Psychiatry 60, 432-435. doi: 10.1016/j.biopsych.2006.02.004

Sklar, P., Ripke, S., Scott, L. J., Andreassen, O. A., Cichon, S., Craddock, N., et al. (2011). Large-scale genome-wide association analysis of bipolar disorder identifies a new susceptibility locus near ODZ4. Nat. Genet. 43, 977-983. doi: $10.1038 /$ ng.943

Smith, D. J., Langan, J., McLean, G., Guthrie, B., and Mercer, S. W. (2013). Schizophrenia is associated with excess multiple physical-health comorbidities but low levels of recorded cardiovascular disease in primary care: cross-sectional study. BMJ Open 3:e002808. doi: 10.1136/bmjopen-2013-00 2808

Sullivan, P. F. (2010). The psychiatric GWAS consortium: big science comes to psychiatry. Neuron 68, 182-186. doi: 10.1016/j.neuron.2010.10.003

Vincent, J., Hovatta, I., Frissa, S., Goodwin, L., Hotopf, M., Hatch, S. L., et al. (2017). Assessing the contributions of childhood maltreatment subtypes and depression case-control status on telomere length reveals a specific role of physical neglect. J. Affect. Disord. 213, 16-22. doi: 10.1016/j.jad.2017.01.031

Whiteford, H. A., Harris, M. G., McKeon, G., Baxter, A., Pennell, C., Barendregt, J. J., et al. (2013). Estimating remission from untreated major depression: a systematic review and meta-analysis. Psychol. Med. 43, 1569-1585. doi: 10.1017/ S0033291712001717

Wikgren, M., Maripuu, M., Karlsson, T., Nordfjäll, K., Bergdahl, J., Hultdin, J., et al. (2012). Short telomeres in depression and the general population are associated with a hypocortisolemic state. Biol. Psychiatry 71, 294-300. doi: 10.1016/j.biopsych.2011.09.015

Winkler, P., Horáček, J., Weissová, A., Šustr, M., and Brunovský, M. (2015). Physical comorbidities in depression co-occurring with anxiety: a cross sectional study in the Czech primary care system. Int. J. Environ. Res. Public Health 12, 15728-15738. doi: 10.3390/ijerph12121 5015

World Health Organisation [WHO] (1993). The ICD-10 Classification of Mental and Behavioural Disorders. Available at: http://apps.who.int/iris/bitstream/ 10665/37108/1/9241544554.pdf

Wray, N. R., Ripke, S., Mattheisen, M., Trzaskowski, M., Byrne, E. M., Abdellaoui, A., et al. (2018). Genome-wide association analyses identify 44 risk variants and refine the genetic architecture of major depression. Nat. Genet. 50, 668-681. doi: 10.1038/s41588-018-0090-3

Yu, W.-Y., Chang, H.-W., Lin, C.-H., and Cho, C.-L. (2008). Short telomeres in patients with chronic schizophrenia who show a poor response to treatment. J. Psychiatry Neurosci. 33, 244-247.

Conflict of Interest Statement: GB has received grant money and acted as a consultant for Eli Lilly.

The remaining authors declare that the research was conducted in the absence of any commercial or financial relationships that could be construed as a potential conflict of interest.

The handling Editor declared a shared affiliation, though no other collaboration, with the authors.

Copyright (c) 2018 Palmos, Breen, Goodwin, Frissa, Hatch, Hotopf, Thuret, Lewis and Powell. This is an open-access article distributed under the terms of the Creative Commons Attribution License (CC BY). The use, distribution or reproduction in other forums is permitted, provided the original author(s) and the copyright owner(s) are credited and that the original publication in this journal is cited, in accordance with accepted academic practice. No use, distribution or reproduction is permitted which does not comply with these terms. 